\title{
$\mathrm{HLA} / \mathrm{RTI}$ 시스템에서 합성전장환경 기반의 \\ 항공 교전 시뮬레이션 모델 구축 프레임워크
}

\author{
함원경 $^{1} \cdot$ 양가람 $^{1} \cdot$ 최종엽 $^{2} \cdot$ 박상철 $^{1 \dagger}$
}

\section{A Framework to Construct the Aviation Engagement Simulation Model based on the Synthetic Battlefield in the HLA/RTI System}

\author{
Won K. Ham $\cdot$ Karam Yang $\cdot$ Jong-Yeob Choi $\cdot$ Sang C. Park
}

\begin{abstract}
This paper proposes a framework to construct the synthetic battlefield based aviation engagement simulation model for the distributed system. The proposed framework has the synthetic battlefield in the HLA (High Level Architecture)/RTI (Run-Time Infrastructure) based distributed system to reflect environmental effects into the aviation engagement simulation model. In an aviation engagement, the environment affects weapon systems such as detection and movement. Therefore, environmental effects are required to be reflected in the simulation. However, former researches are inadequate for complex operations of weapon systems that are requirements of the engagement simulation. Thus, the construction of the engagement simulation system of which reflects environmental effects based on environmental data is still difficult. The main objective of this paper is to propose a framework to solve the difficulty and constructs an example system based on the proposed framework.
\end{abstract}

Key words : Aviation, Engagement Model, Distributed system, HLA/RTI, Simulation, Synthetic Battlefield

\section{요 약}

본 논문에서는 항공 교전 시뮬레이션을 위한 모델을 구축함에 있어서 분산 시스템 상의 합성전장환경 기반으로 구성되는 시스템 구축을 위한 프레임워크를 제안한다. 제안된 프레임워크는 HLA (High Level Architecture)/RTI (Run-Time Infrastructure) 기반의 분산 시스템 상에서 합성전장환경을 구축하여 항공 교전 시뮬레이션 모델에 환경의 영향을 반영하기 위한 시스템을 설계한다. 항공 전투에서 환경은 탐지체계 및 기동체계 등에 영향을 주기 때문에 시뮬레이션에서 그 영향을 반영할 필요가 있다. 그러나 교전급 시뮬레이션에서 요구하는 무기체계의 복합적 운용에 관련된 프레임워크 연구는 기존에 미흡하였고, 이로 인해 환경 데이터를 기반으로 시뮬레이션에 환경 영향을 반영하는 교전급 시뮬레이션 시스템의 구축은 난해 함을 갖는다. 본 논문은 이와 같은 난해함을 해결하기 위한 프레임워크를 제안하고, 제안된 프레임워크를 기반으로 예제 시스템 을 구축하는데 목적을 둔다.

주요어 : 항공, 교전 모델, 분산 시스템, HLA/RTI, 시뮬레이션, 합성전장환경

*본 연구는 본 연구는 방위사업청(UD110006MD), 국방과학연구소(UD100009DD, UD120035JD)의 지원으로 수행되었습니다.

접수일(2012년 12월 12일), 심사일(2013년 4월 30일),

게재 확정일(2013년 12월 17일)

1) 아주대학교 산업공학과

2) 방위사업청 $\mathrm{M} \& \mathrm{~S}$ 기술팀

주 저 자: 함원경

교신저자 : 박상철

E-mail; scpark@ajou.ac.kr

\section{1. 서 론}

모델링 및 시뮬레이션(M\&S, Modeling and Simulation) 을 소요정의, 개념개발, 시제생산, 시험평가, 생산배치, 운 용유지 및 후속 군수지원의 7단계로 구성되는 무기체계 획득의 전 순기(Acquisition Cycle)에 적용하는 $\mathrm{SBA}$ (시 뮬레이션 기반 획득, Simulation-Based Acquisition)에 대한 중요성이 높아지고 있다(Choi, Pyun, 2008). 
국방 분야의 $\mathrm{M \& S}$ 는 표현 정도에 따라 전구, 임무/전 투, 교전, 공학급으로 구분할 수 있는데, 그 중 본 논문의 목적인 교전급 $\mathrm{M \& S}$ 는 몇 분에서 몇 시간 정도의 단일 작전을 대상으로(Hill, McIntyre, 2001) 전투 개체의 행위 와 각 무기체계의 기능을 자세히 묘사하며 적의 위협이나 작전 형태는 부분적으로 모델링한다(Cha, 2007; Hawley, Blauwkamp, 2010). 이와 같은 교전급 M\&S 모델의 특성 상 실제 전장환경의 모의가 요구되는데, 그 이유는 실제 교전의 결과가 환경의 영향에 의해서 달라질 수 있기 때 문이다. 따라서 교전급 M\&S 모델은 합성전장환경을 포 함하여 실제 전장의 환경 영향을 모의할 수 있어야 한다 (ROKSA, 2007; Park et al., 2010).

본 연구의 목적인 항공 교전에서의 핵심 요소인 레이 더는 비교적 환경의 영향으로부터 자유로운 탐지체계로 알려져 있지만, 기존의 걸프전과 코소보전의 결과는 레이 더의 운용도 환경의 영향을 감안해야 한다는 것을 말해주 고 있다(Ban, 2001). 또한 비행체의 기동 성능과 항공 교 전에서 사용되는 다양한 유도방식의 공격체계의 탐지 성 능도 대기 상황과 밀접한 영향을 갖는다 $(\mathrm{Kim}, 2008$; Lim, 2005; Choi, 2008). 따라서 항공 교전을 모의하기 위한 교전급 시뮬레이션 시스템은 합성전장환경 기반으 로 시뮬레이션을 수행하여 환경의 영향을 시뮬레이션 결 과에 반영해야 한다(Kye et al., 2007; Keane et al., 2000).

그러나 실제 시뮬레이션 시스템 구축에 환경의 영향을 반영하는 것은 어려움이 있다. 그 이유는 실제 데이터 제 공자가 제공하는 데이터가 원시 데이터인 환경 수치 데이 터인 반면, 시뮬레이션 시스템에서 필요로 하는 환경 데 이터는 재사용 가능하고 상호 운용성이 있는 데이터라는 상황에서 발생한 격차에 기인한다(Choi, 2008). 즉, 환경 데이터가 존재할 때, 그 데이터를 시뮬레이션에 반입 (Import)하여 시뮬레이션 결과에 반영하기 위해서는 환경 데이터를 사용할 시뮬레이션 엔지니어가 환경에 대해 이 해할 필요가 있다. 하지만 환경에는 해양, 대기, 우주, 지 형, 도심 등 광범위한 스펙트럼이 존재하며, 시뮬레이션 엔지니어가 환경 수치 데이터의 구조를 모델링하여 합성 환경으로 활용하는 것은 난해하며 비효율적인 작업이다. 그러므로 그 격차를 해결해 줄 중간 프로세스가 요구된다 (Son, 2003; Hwam et al., 2012).

본 논문에서는 이와 같은 격차를 해결하기 위한 프로 세스로 항공 교전 시뮬레이션 시스템의 구축 프레임워크 를 제안한다. 제안되는 프레임워크는 HLA(High Level Architecture)/RTI(Run-Time Infrastructure) 기반의 분산 시스템에서 항공 교전을 위한 합성전장환경을 구축하고,
이를 통해 환경의 영향을 시뮬레이션 모델에 반영한다. 기존에 연구들은 단위 무기체계에 환경의 영향을 반영하 는 것이 중심이 되며(Lee et al., 2007) 교전급 시뮬레이 션에서 요구하는 무기체계의 복합적 운용에 관련된 프레 임워크 연구는 미흡하다.

이후 논문의 구성은 다음과 같다. 2장에서 본 논문에서 제안되는 프레임워크의 전반적인 접근방법을 소개하고, 3 장에서 제안된 시뮬레이션 프레임워크의 구성을 설명한 다. 4장에서는 제안된 프레임워크를 기반으로 구축된 예 제 시뮬레이션 시스템을 소개하며 마지막으로 5장에서 결 론을 맺는다.

\section{2. 접근방법}

\subsection{HLA/RTI 기반의 분산 시뮬레이션 시스템}

$\mathrm{HLA} / \mathrm{RTI}$ 시스템은 분산 시스템 설계 방법 중 게시-구 독(P-S, Publish- Subscribe) 방법으로 데이터를 교환하는 간접 커뮤니케이션 패러다임을 따른다. P-S 시스템은 서 버와 클라이언트로 구성되며, 클라이언트들은 자신에게 할당된 데이터들을 서버의 특정부분에 게시하고, 필요한 데이터들을 서버의 특정부분에서 구독하면서 통신한다. 이와 같은 시스템은 사건 기반의 분산 시스템의 구성에 효과적인 방법이다(Coulouris et al., 2012).

HLA/RTI 기반 시스템에서는 클라이언트를 페더레이 트라 칭하며, 페더레이트들이 RTI 서버에 데이터를 게시 하고 구독한다. 이와 같은 전체 시뮬레이션 시스템을 페 더레이션(Federation)이라 하며, 각 페더레이트(Federate) 들이 시뮬레이션 수행 중 교환할 데이터 내역들을 사전에 정의한 문서를 페더레이션 객체 모델(FOM, Federation Object Model)이라 한다. FOM은 객체 모델 서식(OMT, Object Model Templates)을 따라서 작성된다. 페더레이 트에서 RTI 서버로부터 FOM의 내용을 게시/구독하기 위 해서 페더레이트는 RTI 인터페이스로 시뮬레이션 객체 모델(SOM, Simulation Object Model)을 갖는다. RTI 서 버와 페더레이트 사이에서 교환되는 데이터 형식은 객체 (Object) 클래스와 인터랙션(Interaction) 클래스가 존재 하는데, 객체 클래스는 지속적으로 공유되는 데이터, 인터 랙션클래스는 메시지와 같이 단발적인 데이터 교환에 적 합하다. 관련된 객체 와 인터랙션 클래스 데이터의 형식은 FOM에 선언되어 있으며, 객체클래스에는 속성(Attribute) 들을, 인터랙션 클래스에는 파러미터(Parameter)들을 하 위에 가져 상세 데이터 목록이 정의 되어있다(Australian Defense Simulation Office, 2004; IEEE Std 1516TM, 
2010).

연구에서는 HLA/RTI 시스템에서 합성전장환경 기반 의 시뮬레이션을 수행하기 위해 항공 교전 모델은 환경 데이터 관리의 역할을 담당하는 환경 페더레이트를 갖는 다. 환경 페더레이트는 항공 전투 시뮬레이션의 환경 데 이터 요구를 구독(Subscribe)하고, 환경 데이터 요구 내용 에 적합한 환경 데이터를 게시(Publish)하여 합성전장환 경을 구축한다. 본 연구에서는 이와 같은 HLA/RTI 시스 템에서 합성전장환경의 구축을 통해 앞서 언급된 환경 데 이터와 데이터 사용자의 요구의 격차를 해결한다.

\section{2 항공 교전을 위한 합성전장환경}

항공 교전을 위한 합성전장환경의 구축은 항공 무기체 계에 영향을 주는 환경 요소인 EPO(Environmental Process Object)를 도출하고, 이에 적합하게 수집된 대기 및 기상 환경 데이터를 기반으로 환경 페더레이트가 환경 데이터 를 게시하는 것으로 수행된다. 페더레이션을 구성하는 다 른 페더레이트들은 필요한 환경데이터를 RTI 서버로부터 구독하여 시뮬레이션에 환경의 영향을 반영한다. Fig. 1 은 이와 같은 시뮬레이션 시스템의 개념도를 표현하고 있 다. Fig. 1 은 항공 전장환경을 분석하고, 분석을 통해 도 출된 $\mathrm{EPO}$ 를 원시 환경데이터 제공자로부터 획득한다. 시 뮬레이션 시스템은 환경 페더레이트에 시뮬레이션에 필 요한 환경 데이터를 요구하고, 환경 페더레이트는 적합한 데이터를 추출하여 제공한다. 시스템은 획득한 환경 데이 터를 이용해 탐지확률 등을 도출하고, 도출된 결과를 시 뮬레이션의 수행에 반영한다. 따라서 시스템은 탐지확률
을 도출하는 페더레이트와 실제 시뮬레이션을 진행하는 페더레이트가 필요하다.

\section{3. 시뮬레이션 프레임워크}

항공 교전 시뮬레이션을 위한 페더레이션에서 각 페더 레이트들은 페더레이션에 연동되어 각각의 다른 어플리 케이션으로 작업을 수행하지만, 전체 시스템의 관점에서 는 하나의 기능을 담당한다. 본 연구에서는 시뮬레이션 페더레이트, 환경 페더레이트 및 환경 데이터와 시뮬레이 션 데이터를 기반으로 레이더 탐지확률을 도출하는 레이 더 탐지체계 페더레이트를 갖는 페더레이션 구축 방안을 제안하며, Fig. 2는 제안된 프레임워크에 기반한 페더레 이션의 구성을 표현한다. 제안된 프레임워크를 통해 시뮬 레이션 엔지니어가 요구하는 환경 데이터에 대해서 환경 엔지니어가 데이터를 공급하고, 공급된 환경 데이터를 활 용하여 전자기파 감쇄가 레이더 특성에 따라 탐지 성능에 미치는 영향을 반영한 레이더 탐지확률을 레이더 엔지니 어가 도출한다. 시뮬레이션 엔지니어는 항공 교전 모델의 시뮬레이션 개체들 사이의 탐지 상태에 대해 레이더 엔지 니어에 의해 도출된 탐지확률을 활용한다.

시뮬레이션의 수행 중 레이더의 전자기파의 전파와 환 경 효과에 의한 전파 감쇄를 계산해서 탐지확률을 도출하 는 과정은 해당 분야의 전문적인 지식이 요구된다. 일반 적으로, 시뮬레이션 엔지니어는 이와 같은 전문 지식을 갖고 있지 않으므로 해당 분야 전문가의 협조가 요구된다. 본 연구에서 제안되는 프레임워크는 시뮬레이션 분야와

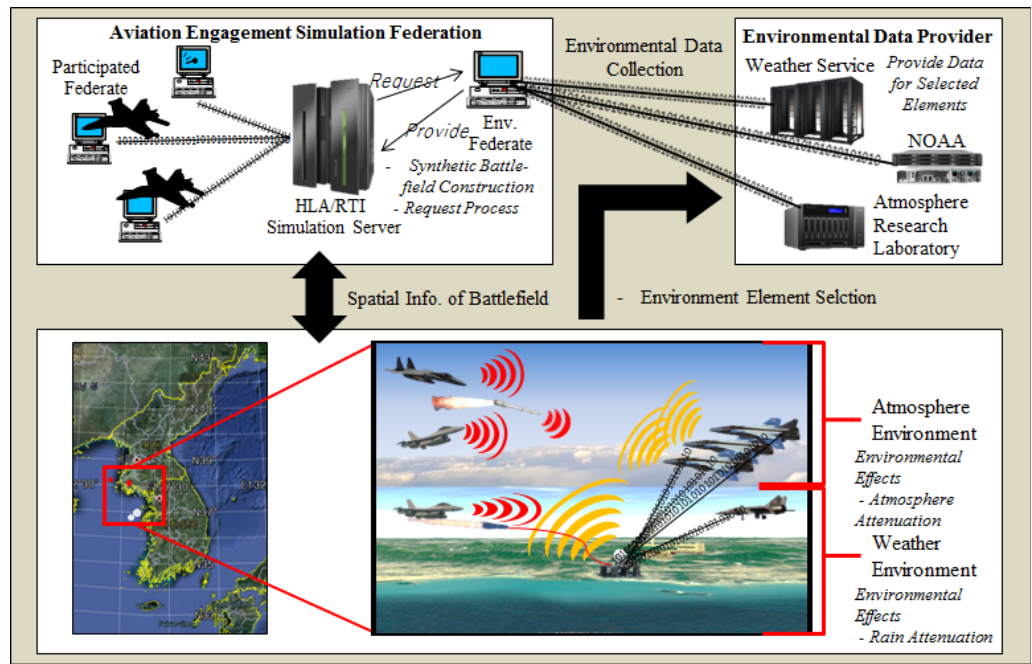

Fig. 1. The Conceptual Simulation System 


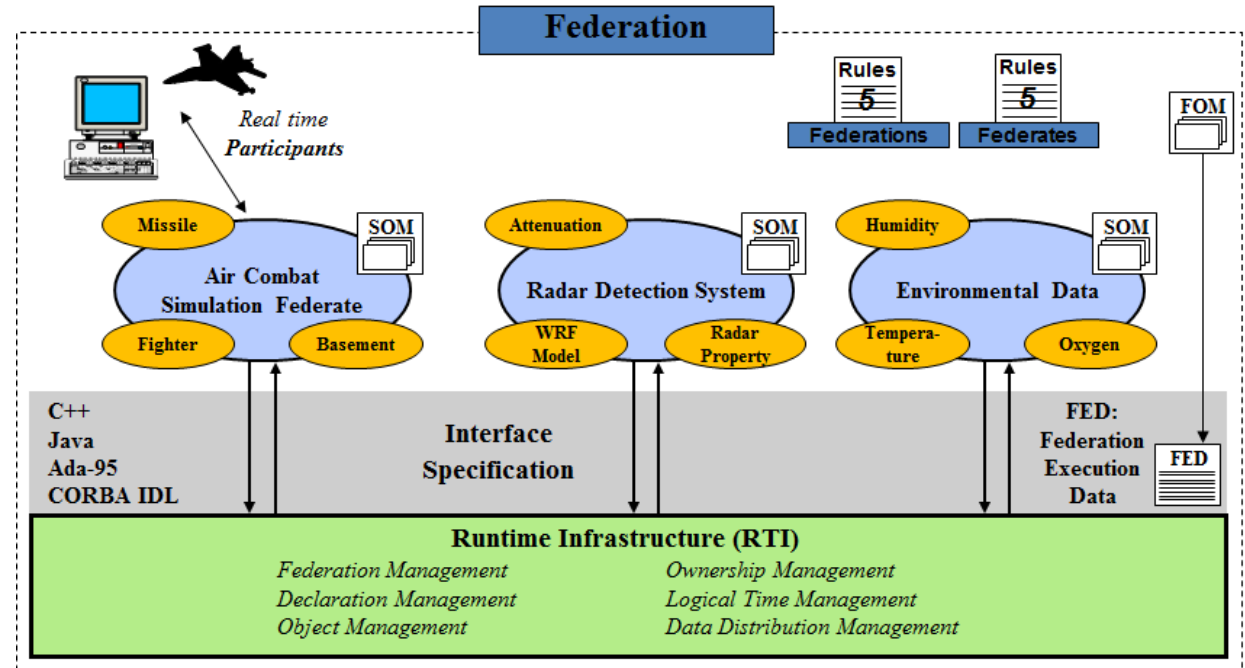

Fig. 2. The Proposed Simulation System in HLA/RTI

\begin{tabular}{|c|c|c|c|}
\hline Distance & GP & P & F \\
\hline Detection Area & $\begin{array}{c}\text { Radar Capability }+ \\
\text { Enviromment }\end{array}$ & $\begin{array}{c}\text { Detection Probability in } \\
\text { Each Detection Area }\end{array}$ & $\begin{array}{c}\text { Cumulative Detection } \\
\text { Probability }\end{array}$ \\
\hline
\end{tabular}

Fig. 3. Glimpse Probability Table

각 전문 분야를 분리시키고, 개별적인 페더레이트의 개발 을 통해 시뮬레이션 시스템 구축에 있어서 각 엔지니어의 역할 분담을 명확히 하고자 한다. 이를 통해 대형 시뮬레 이션 시스템의 구축에서 발생하는 기능 및 역할 분류의 모호함을 해결하고, 동시 공학적인 페더레이트의 개발로 효율적인 항공 교전 시뮬레이션 시스템 구축이 가능하다.

본 연구에서는 시뮬레이션 개체 사이에서 탐지확률의 반영을 돕기위해 GP(Glimpse Probability) 테이블의 개 념을 도입하였다. GP 테이블은 두 개의 열로 구성되며, 첫 째 열은 거리 범위를 표현하고 둘째 열은 해당 거리 범위 에서의 GP를 표현한다(Korea military academy, 2004). $\mathrm{GP}$ 는 특정 거리범위에서 레이더의 탐지 성능을 의미하 며, 레이더 탐지 성능에 환경 영향을 반영하여 계산된다. Fig. 3의 좌측 두 개열은 GP 테이블이며, GP 테이블을 이용해서 우측 두 개열인 해당 영역에서의 탐지확률 $\mathrm{P}$ 와 누적 탐지확률 $\mathrm{F}$ 를 구하여 시뮬레이션에 반영할 수 있음 을 표현한다. $\mathrm{P}$ 는 식 (1)과 같이 도출되며, $\mathrm{F}$ 는 식 (2)와 같이 도출된다. 첫 거리범위에서 $\mathrm{F}$ 가 1 을 만족하는 거리 범위까지의 거리범위들 사이에서 개체의 탐지가 기대된 다(Driels, 2004).

$$
\begin{aligned}
& \mathrm{P}_{\mathrm{n}}=\left(1-\mathrm{GP}_{1}\right) \cdot \cdots \cdot\left(1-\mathrm{GP}_{\mathrm{n}-1}\right) \cdot \mathrm{GP}_{\mathrm{n}} \\
& \mathrm{F}_{\mathrm{n}}=\sum_{k=1}^{n} \mathrm{P}_{\mathrm{k}}
\end{aligned}
$$

합성환경을 반영하는 항공 교전 시뮬레이션 모델 구축 을 위해 제안된 본 연구의 프레임워크는 HLA/RTI 기반 의 시뮬레이션 시스템에서 환경과 레이더의 탐지 성능을 반영한 GP 테이블을 적용하여 구성되며, Fig. 4는 프레임 워크에서 페더레이션 구성과 페더레이트의 운용과 페더 레이트 사이의 데이터 교환을 표현하고 있다. Fig. 4에서 표현하는 페더레이트 운용과 데이터 교환의 절차는 다음 에 설명되어 있으며, 같은 번호는 동시에 발생함을 의미 한다. HLA/RTI 시스템의 특성상 모든 데이터 교환은 RTI 서버를 경유하여 발생하며, 다음의 설명에서 페더레 이트가 데이터를 서버에 게시하고 해당되는 페더레이트 가 데이터를 구독하는 과정을 전송이라고 표현하였다.

1) 시뮬레이션 페더레이트는 전장공간의 시간, 위도, 경 도 및 높이/깊이 범위(Range)와 해상도(Resolution)를 환경 페더레이트에 전송한다.

$\left.1^{\prime}\right)$ 시뮬레이션 페더레이트는 위도, 경도 및 높이/깊이로 이루어진 레이더 위치 정보와 출력, 주파수 등의 레이 더 특성정보(Radar Spec.), 그리고 비어있는 GP 테이 블을 레이더 탐지체계 페더레이트에 전송한다. 


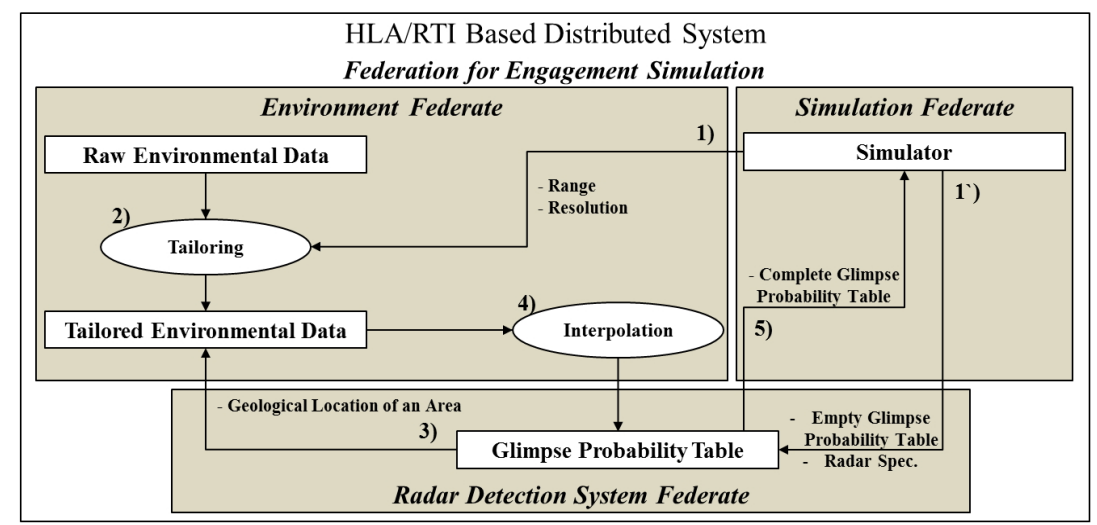

Fig. 4. Simulation System of the Proposed Framework

2) 환경 페더레이트는 보유한 환경 데이터(시간, 위도, 경 도, 높깊이에 따른 기온, 습도, 기압, 강수량, 자기장 등의 속성)에서 전장공간 범위에 해당하는 데이터를 추출(Tailoring)하여 메모리에 반입한다.

3) 탐지체계 페더레이트는 수신한 레이더의 위치 정보를 환경 페더레이트에 전송한다.

4) 환경 페더레이트는 레이더의 위치 정보에 해당하는 데 이터(기온, 습도, 기압, 강수량, 자기장 등)를 추출된 데이터에서 보간법(Interpolation)을 통해 도출한 뒤 레이더 탐지체계 페더레이트에 전송한다.

5) 레이더 탐지체계 페더레이트는 환경 데이터와 레이더 특성정보를 활용해 전파의 전파에 관련된 공학급 모델 의 결과를 도출하여 거리에 따른 탐지확률을 GP 테이 블에 삽입하여 완성하고 시뮬레이션 페더레이트에 전 송한다.

최종적으로 완성된 GP 테이블을 구독한 시뮬레이션 페더레이트는 앞서 언급된 $\mathrm{P}$ 와 $\mathrm{F}$ 를 계산하고, 그 결과를 항공 교전 시뮬레이션의 수행 중 시뮬레이션 개체들 사이 의 탐지상태에 적용한다.

제안된 프레임워크에서 환경 엔지니어는 환경 페더레 이트, 레이더 엔지니어는 레이터 탐지 페더레이트, 마지막 으로 시뮬레이션 엔지니어는 시뮬레이션 페더레이트의 구축 및 운용을 통해 합성전장환경 기반의 항공 교전 시 뮬레이션을 수행한다.

\section{4. 프레임워크 구현}

본 연구에서 제안한 프레임워크를 기반으로 항공 교전
을 위한 예제 시뮬레이션 시스템을 구축하였다. 예제 시 스템은 제안된 프레임워크의 페더레이션 설계에 따라 시 뮬레이션 페더레이트, 레이더 탐지체계 페더레이트 및 환 경 페더레이트로 구성되어 있다. 환경 페더레이트는 대기 환경 데이터를 가지며, 시뮬레이션 페더레이트가 게시한 시간, 위도 및 경도에 따른 전장공간의 범위의 환경데이 터를 추출한다. 시뮬레이션 페더레이트는 수상함에서 항 공기를 공격하는 간단한 시나리오에 대해 시뮬레이션을 수행한다. 예제 시나리오는 항공기들이 $\mathrm{A}$ 지점에서 생성 되고, 수상함이 위치한 공역을 지나 B 지점으로 이동한다. 이동 중 수상함이 GP 테이블의 탐지확률에 의해 항공기 를 탐지하면 미사일을 발사하여 격추시키고, 탐지하지 못 하였을 경우 항공기가 B 지점에 도착한다. 시뮬레이션 페 더레이트는 수상함이 갖는 레이더 특성 정보와 위치 정보 를 포함하는 비어있는 GP 테이블을 게시한다. 레이더 탐 지체계 페더레이트는 수상함이 게시한 정보를 구독하여 현재 수상함이 위치와 관계된 환경 데이터 요구를 게시한 다. 환경 페더레이트는 요구에 따른 환경 데이터를 게시 하고, 탐지체계 페더레이트는 게시된 환경 데이터와 레이 더 특성을 파러미터로 탐지확률을 계산하여 GP 테이블을 완성한다. 완성된 GP 테이블은 RTI 서버에 게시되고, 시 뮬레이션 페더레이트는 GP 테이블을 구독하여 수상함의 항공기 요격 성공률에 반영한다.

예제 시뮬레이션 시스템은 Pitch사의 pRTI1516을 사 용하여 HLA/RTI 시스템을 구축하였고, C++ 기반의 Ogre3D 가시화 엔진을 사용하여 시뮬레이션 페더레이트를 구축 하였다. 환경 페더레이트는 강수량을 $\mathrm{EPO}$ 로 도출하고, 기상청 데이터베이스의 강수량 데이터를 사용하였다. 시 뮬레이션 페더레이트에서 레이더 특성 정보로는 레이더 
출력과 주파수를 가지며, 레이더 탐지 페더레이트는 탐지 성능을 출력에 비례하고 주파수와 강수량에 반비례하도록 하여 데모 레이더 탐지 성능을 도출하도록 하였다. Fig. 5

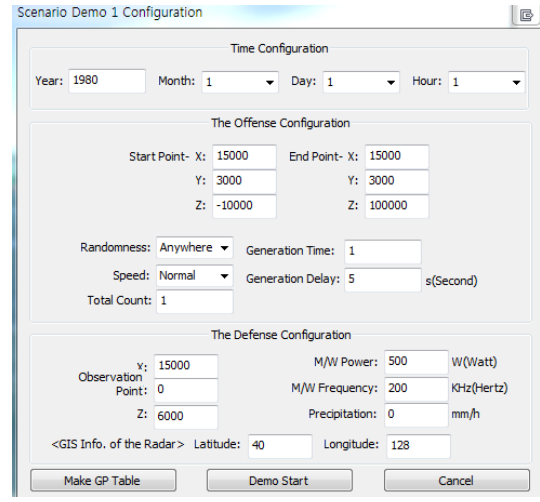

Fig. 5. Scenario Configuration Dialog

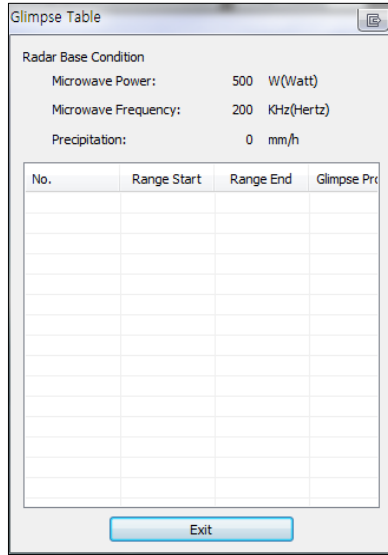

(a) Empty GP Table

\begin{tabular}{|c|c|c|c|c|}
\hline Glimpse & able & & & 国 \\
\hline Radar & ise Condition & & & \\
\hline & owave Power: & 500 & w(Watt) & \\
\hline & owave Frequency & 200 & $\mathrm{~Hz}($ Hertz) & \\
\hline & cipitation: & 25 & $\mathrm{~mm} / \mathrm{h}$ & \\
\hline No. & Range Start & Range End & Glimpse Prob. & A \\
\hline 0 & 4900.000000 & 5000.000000 & 7.367011 & \\
\hline 1 & 4800.000000 & 4900.000000 & 1.435132 & \\
\hline 2 & 4700.000000 & 4800.000000 & 1.339457 & \\
\hline 3 & 4600.000000 & 4700.000000 & 1.913509 & \\
\hline 4 & 4500.000000 & 4600.000000 & 5.453502 & \\
\hline 5 & 4400.000000 & 4500.000000 & 9.376196 & \\
\hline 6 & 4300.000000 & 4400.000000 & 6.505932 & \\
\hline 7 & 4200.000000 & 4300.000000 & 5. 166475 & \\
\hline 8 & 4100.000000 & 4200.000000 & 8.323766 & \\
\hline 9 & 4000.000000 & 4100.000000 & 0.000000 & \\
\hline 10 & 3900.000000 & 4000.000000 & 4.209721 & \\
\hline 11 & 3800.000000 & 3900.000000 & 8.706468 & \\
\hline 12 & 3700.000000 & 3800.000000 & 0.000000 & \\
\hline 13 & 3600.000000 & 3700.000000 & 8.323766 & \\
\hline 14 & 3500.000000 & 3600.000000 & 4.879449 & - \\
\hline
\end{tabular}

(b) Complete GP Table

Fig. 6. GP Table Generation

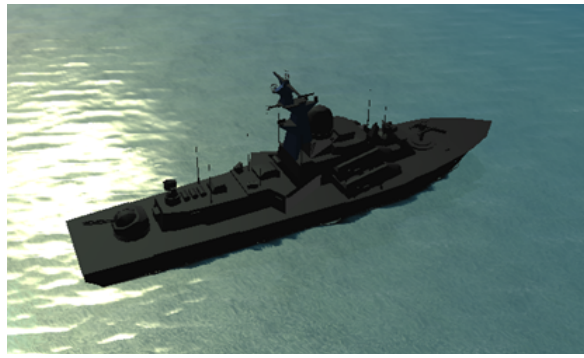

(a) The Surface Ship

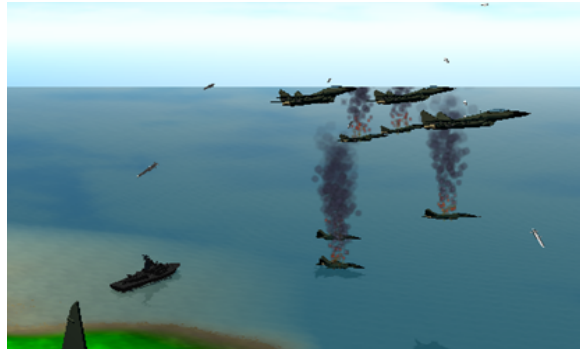

(b) The Aviation Engagement Simulation

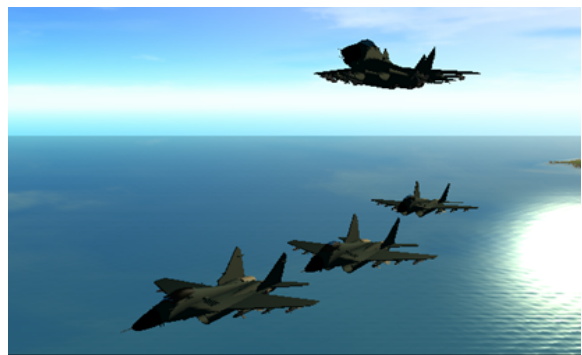

(b) Complete GP Table

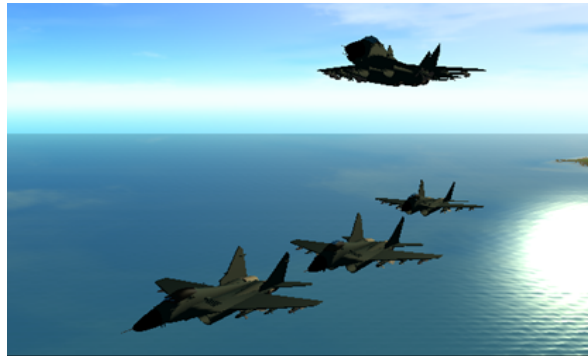

(c) Detected and Destroyed Aircraft

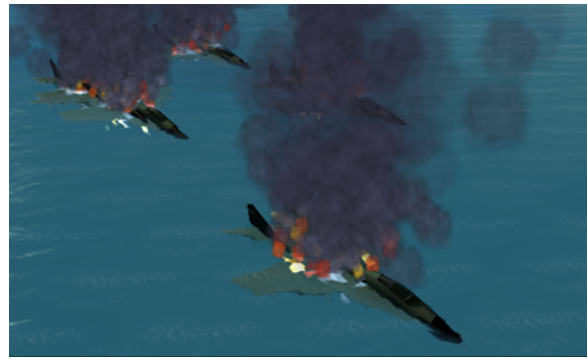

(d) Undetected and Survived Aircraft

Fig. 7. The Result of the Simulation of the Example System 
는 시뮬레이션 페더레이트에서 시뮬레이션 수행을 위한 시나리오를 설정하는 UI(User Interface)로서, 수상함의 위치와 항공기들의 생성 지점 및 도착 지점, 생성 대수, 횟수 및 레이더의 출력과 주파수 등을 설정한다.

시나리오 설정이 완료되면 Fig. 6. (a)에 표현된 비어있 는 GP 테이블을 생성하고, 해당되는 정보를 레이더 탐지 체계 페더레이트가 구독할 수 있도록 게시한다. 레이더 탐지체계 페더레이트에 의해 완성된 GP 테이블은 시뮬레 이션 페더레이트에 의해 Fig. 6. (b)와 같이 구독된다.

완성된 GP 테이블을 기반하여 수상함이 항공기를 탐 지할 확률을 도출하고 시뮬레이션을 수행한다. 시뮬레이 션 수행 및 결과는 Fig. 7에 표현되었다.

\section{5. 결론 및 향후연구}

합성전장환경을 기반으로 교전 시뮬레이션의 수행에 환경의 영향을 반영하는 것은 차세대 국방 $\mathrm{M} \& \mathrm{~S}$ 에서 핵 심적인 요소로 언급되어왔다. 그러나 교전급 시뮬레이션 모델의 합성전장환경 기반 구축을 위한 기존의 연구는 미 흡한 수준이며, 특히, 합성전장환경을 구축하여 시뮬레이 션에 환경의 영향을 반영하는 방법론에 관련된 연구는 매 우 부족하다. 본 연구에서는 항공 교전에 대한 교전급 시 뮬레이션 모델의 구축에 합성전장환경의 구축 및 시뮬레 이션에 반영을 위한 환경 데이터의 공급, 활용 및 시뮬레 이션 적용 방안을 설계한 프레임워크를 제안하였다. 제안 된 프레임워크는 HLA/RTI 분산 시스템을 기반으로 구성 되며, 페더레이션을 구성하는 페더레이트들이 하나의 전 문 분야를 담당함으로서 서론에서 언급한 격차를 해결할 수 있다. 본 연구에서는 제안된 프레임워크에 기반한 예 제 항공 교전 시뮬레이션 시스템을 구축하여 시뮬레이션 을 수행하였고, 이를 통해서 프레임워크의 목적 달성을 확인할 수 있었다.

향후연구로는 환경의 영향을 반영함에 있어서 탐지 확 률의 도출에 한정되지 않고, 항공기의 기동, 유도 무기의 정확성 및 기만 가능성 등을 도출할 수 있는 페더레이션 의 구축을 수행한다.

\section{References}

1. Australian Defense Simulation Office (2004), Distributed simulation guide, Department of Defence, Canberra, Australia.

2. K.S. Ban (2001), Weather and war, Myunjin publishing
Co., Korea.

3. J.S. Cha (2007), "Application of defense M\&S", Journal of IEEK 25(11), pp. 102-108.

4. S.Y. Choi (2008), "Future and Technology Development of Defense Modeling and Simulation“, The magazine of the IEEK 35(10), pp. 1157-1166.

5. S.Y. Choi and J.J. Pyun (2008), "Development Outlook and Concepts of SBA(Suimulation Based Acquisition)", Journal of KIISE 26(11), pp. 6-12.

6. Coulouris, G., Dollimore, J., Kindberg, T., Blair, G. (2012), Distributed systems: Concepts and design, fifth ed, Addison-Wesley, USA.

7. Driels, M. (2004), Weaponeering: Conventional Weapon System Effectiveness, first ed, American Institute of Aeronautics and Astronautics, USA.

8. Hawley, P. A., Blauwkamp, R. A. (2010), “ Six-Degreeof-Freedom digital simulations for missile guidance, navigation, and control”, Johns hopkins APL technical digest, 29(1), pp. 71-84.

9. W.K. Hwam, J-H. Kim, Y-N. Na, S-U. Cheon and S.C. Park (2012), "Generation of the battlefield in distributed simulation system based on synthtetic environment representation and interchange standard (SEDRS)“, Journal of Information Technology and Architecture 9(3), pp. 253-263.

10. Hill, Raymond R., McIntyre, Gregory A. (2001), "Applications of Discrete Event Simulation Modeling To Military Problems", Proceedings of the 2001 winter simulation conference.

11. IEEE Std 1516TM (2010), IEEE Standard for Modeling and Simulation (M\&S): High Level Architecture (HLA) - Framework and rules (2010 Revised Ed), IEEE Computer Society, New York, USA.

12. Keane, J. F., Lutz, R. R., Myers, S. E. and Coolahan, J. E. (2000), "An Architecture for Simulation Based Acquisition", Johns Hopkins APL Technical Digest, 21(3), pp. 348-358.

13. Kim DI (2008), The solution and measuring technology of microwave interference, Honglung science publishing Co., Korea.

14. Korea Military Academy. (2003). Weapons System Engineering, Bookshill, Korea.

15. J.S. Kye, W.S. Kim and S.J. Kang (2007), "Synthetic Environment Modeling Methodology for National Defense Modeling and Simulation", Journal of IEEK 25(11), pp. 32-38.

16. I.K. Lee, D.C. Park, T.S. Yoo, R.H. Myung, J.S. Lim, Y.H. Cho and H.C. Kang (2006). Radar Engineering and Applications in Electronic Warfare, Daeyoung, Seoul. 
17. S.M. Lim (2005), Understanding of combat air plane, Easy book, Korea.

18. Sang C. Park, Kwon, Yongjin, Seong, Kilyeong and Pyun, Jaijeong (2010), "Simulation Framework for Small Scale Engagement", Computer\&Industrial Engineering, 59, pp. 463-472.
19. Park SC, Seong KY (2010), "A synthetic environment based engagement simulation model", Transactions of the Society of CAD/CAM engineers 15(4), pp. 271-278.

20. ROKSA (2007), History of War, Global Books, Korea.

21. Son ME (2003), "Introduction to SEDRIS", Research of defense policy 59, pp. 99-127.

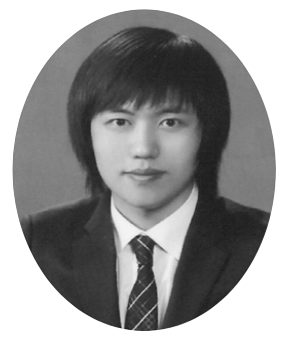

함 원 경 (lunacy@ajou.ac.kr)

2011 아주대학교 산업정보시스템공학부 학사

2011 2013 아주대학교 대학원 산업공학과 석사

2013 현재 아주대학교 대학원 산업공학과 박사과정

관심분야 : 국방 $\mathrm{M \& S}$, 분산 시뮬레이션, 합성전장환경, $3 \mathrm{D}$ 가시화

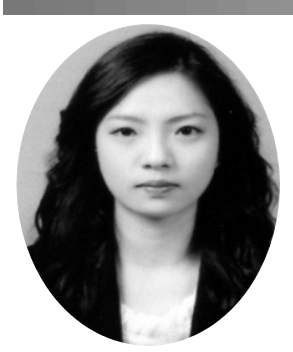

양 가 람 (vision0429@ajou.ac.kr)

2011 동양대학교 철도차량학부 학사

2012 현재 아주대학교 대학원 산업공학과 석사과정

관심분야 : 국방 $\mathrm{M \& S}$, 분산 시뮬레이션, 합성전장환경

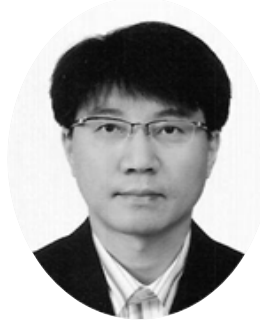

최 종 엽 (jmerit@kg21.net)

2005 아주대학교 대학원 정보통신학과 공학석사

2012 광운대학교 대학원 방위사업학과 박사과정 수료

2006 현재 방위사업청 $\mathrm{M} \& \mathrm{~S}$ 기술팀 사무관

관심분야 : 국방M\&S, 합성전장환경, HLA/RTI

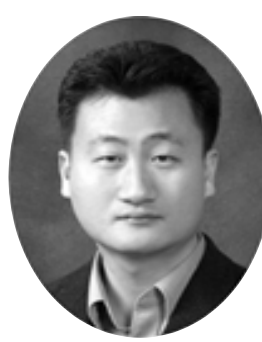

박 상 철 (scpark@ajou.ac.kr)

Ph.D. (2000) in Industrial Engineering, Dept of I.E., KAIST, Korea

B.S. (1994) in Industrial Engineering, Dept of I.E., KAIST, Korea

M.S. (1996) in Industrial Engineering, Dept of I.E., KAIST, Korea

2000년 9월 2001년 12월 큐빅테크, 선임연구원

2002월 1년 2004년 2월 DaimlerChrysler ITM Dept. Research Engineer

2008년 2월 현재 아주대학교 산업정보시스템 공학부, 교수

관심분야 : 시뮬레이션, 제조 시스템, 이산사건 모델링, $\mathrm{CAD} / \mathrm{CAM}, \mathrm{PLC}$ 